ANNALES

POLONICI MATHEMATICI

$83.1(2004)$

\title{
A proof of the Livingston conjecture for the fourth and the fifth coefficient of concave univalent functions
}

\author{
by KARL-JOACHIM WiRThS (Braunschweig)
}

\begin{abstract}
Let $D$ denote the open unit disc and $f: D \rightarrow \overline{\mathbb{C}}$ be meromorphic and injective in $D$. We further assume that $f$ has a simple pole at the point $p \in(0,1)$ and an expansion

$$
f(z)=z+\sum_{n=2}^{\infty} a_{n}(f) z^{n}, \quad|z|<p .
$$

In particular, we consider $f$ that map $D$ onto a domain whose complement with respect to $\overline{\mathbb{C}}$ is convex. Because of the shape of $f(D)$ these functions will be called concave univalent functions with pole $p$ and the family of these functions is denoted by $\operatorname{Co}(p)$. It is proved that for $p \in(0,1)$ the domain of variability of the coefficient $a_{n}(f), f \in \operatorname{Co}(p)$, for
\end{abstract} $n \in\{2,3,4,5\}$ is determined by the inequality

$$
\left|a_{n}(f)-\frac{1-p^{2 n+2}}{p^{n-1}\left(1-p^{4}\right)}\right| \leq \frac{p^{2}\left(1-p^{2 n-2}\right)}{p^{n-1}\left(1-p^{4}\right)} .
$$

In the said cases, this settles a conjecture from [1]. The above inequality was proved for $n=2$ in [6] and [2] by different methods and for $n=3$ in [1]. A consequence of this inequality is the so called Livingston conjecture (see [4])

$$
\operatorname{Re}\left(a_{n}(f)\right) \geq \frac{1+p^{2 n}}{p^{n-1}\left(1+p^{2}\right)} .
$$

A classical problem in Geometric Function Theory is to determine the domain of variability of the $n$th Taylor coefficient for classes of functions $f$ holomorphic and univalent in the unit disc $D$ and determined by the shape of $f(D)$. Famous examples are the classes of convex or starlike functions. In many of these cases the relevant families are invariant under rotations around the origin and therefore the domains of variability under consideration are discs with center at the origin.

In the present paper we consider a family of meromorphic mappings which is not invariant under rotations and we will determine the domain

2000 Mathematics Subject Classification: 30C50, 30C45.

Key words and phrases: concave univalent functions, domain of variability, Taylor coefficient. 
of variability of its Taylor coefficient $a_{n}(f), n \in\{2,3,4,5\}$, by a unified method. We are concerned with the family of concave univalent functions with pole $p \in(0,1)$, denoted by $\operatorname{Co}(p)$. We say that a function $f: D \rightarrow \overline{\mathbb{C}}$ belongs to $\mathrm{Co}(p)$ if:

(i) $f$ is meromorphic in $D$ and has a simple pole at $p \in(0,1)$.

(ii) $f$ has an expansion

$$
f(z)=z+\sum_{n=2}^{\infty} a_{n}(f) z^{n}, \quad|z|<p .
$$

(iii) $f$ maps $D$ conformally onto a set whose complement with respect to $\overline{\mathbb{C}}$ is convex.

The story of the coefficients of concave functions began with J. Miller's proof of the inequality

$$
\left|a_{2}(f)-\frac{1+p^{2}+p^{4}}{p\left(1+p^{2}\right)}\right| \leq \frac{p}{1+p^{2}}
$$

for functions $f$ that satisfy (i), (ii) and

$$
\operatorname{Re}\left(1+\frac{z f^{\prime \prime}(z)}{f^{\prime}(z)}+\frac{2 p}{z-p}-\frac{2 p z}{1-p z}\right)<0, \quad z \in D
$$

(see [6]). In fact, at this time Pfaltzgraff and Pinchuk had essentially proved in [7] that the class of functions that satisfy this set of conditions equals $\mathrm{Co}(p)$. This fact was recognized by A. E. Livingston in the article [4] published in these Annales. In Livingston's paper, which mainly motivated the research presented here, it was proved that

$$
\operatorname{Re}\left(a_{3}(f)\right) \geq \frac{1+p^{6}}{p^{2}\left(1+p^{2}\right)}
$$

for $f \in \operatorname{Co}(p), p \in(0,1)$. The extremal functions for (1) and (3) are

$$
f_{\theta}(z)=\frac{z-\frac{p}{1+p^{2}}\left(1+e^{i \theta}\right) z^{2}}{(1-z / p)(1-z p)},
$$

where $\theta \in[0,2 \pi)$ for (1) and $\theta=0$ for (3). The form (4) of the extremal functions led Livingston to the conjecture that

$$
\operatorname{Re}\left(a_{n}(f)\right) \geq \frac{1+p^{2 n}}{p^{n-1}\left(1+p^{2}\right)}
$$

for all $n \geq 2, f \in \operatorname{Co}(p), p \in(0,1)$. Similar considerations led to a sharper version of this conjecture, namely

$$
\left|a_{n}(f)-\frac{1-p^{2 n+2}}{p^{n-1}\left(1-p^{4}\right)}\right| \leq \frac{p^{2}\left(1-p^{2 n-2}\right)}{p^{n-1}\left(1-p^{4}\right)}
$$


for all $n \geq 2, f \in \operatorname{Co}(p), p \in(0,1)$, which generalizes (1) (see [1]). The case $n=3$ of this conjecture was proved in [1].

Concerning (5) it was proved in [3] that

$$
\operatorname{Re}\left(a_{n}(f)\right) \geq \frac{1+p^{2 n}}{p^{n-1}(1+p)^{2}}
$$

for all $n \geq 2, f \in \operatorname{Co}(p), p \in(0,1)$.

In the present paper we prove a new representation formula for $f^{\prime \prime} / f^{\prime}$, $f \in \mathrm{Co}(p)$, which enables us to give a unified proof of (6) for $n \in\{2,3,4,5\}$. Because of the simplicity of this proof we want to express the hope that someone among the interested readers will see a generalization of the formulae given below that will prove the conjecture (6) for all $n \geq 2$.

Theorem 1. A function $f$ belongs to the class $\mathrm{Co}(p)$ if and only if $f$ satisfies (ii) and there exists a function $\omega$ holomorphic in $D$ such that $\omega(D) \subset \bar{D}$ and

$$
\frac{f^{\prime \prime}(z)}{f^{\prime}(z)}=\frac{2 p}{1-z p}+\frac{2}{p(1-z / p)}+\frac{2 z \omega(z)-\alpha(1+\omega(z))}{1-\alpha z(1+\omega(z))+z^{2} \omega(z)}, \quad z \in D,
$$

where

$$
\alpha:=\frac{2 p}{1+p^{2}} \in(0,1) .
$$

Proof. We will give two short alternative proofs of this theorem. One of them starts with formula (2) and the other one with the following representation formula:

A function $f$ belongs to the class $\operatorname{Co}(p)$ if and only if $f$ satisfies (ii) and there exists a function $\psi$ holomorphic in $D$ such that $\psi(D) \subset \bar{D}$ and

$$
\frac{2 f^{\prime}(z)}{f^{\prime \prime}(z)}+z=\frac{p+\left(\frac{z-p}{1-z p}\right)^{3} \psi(z)}{1+\left(\frac{z-p}{1-z p}\right)^{3} p \psi(z)}, \quad z \in D
$$

(see [1] and [2]). Hence, by this procedure we get a short proof for the equivalence of (2) and (8).

On the one hand, the considerations in [5], [7] and [4] concerning (2) together with a little computation show that $f \in \operatorname{Co}(p)$ if and only if $f$ satisfies (ii) and there exists a function $P$ holomorphic in $D$ such that

$$
\operatorname{Re}(P(z))>0 \quad \text { for } z \in D, \quad P(p)=\frac{1+p^{2}}{1-p^{2}}, \quad P(0)=1
$$

and

$$
\frac{f^{\prime \prime}(z)}{f^{\prime}(z)}=\frac{2 p}{1-z p}+\frac{2}{p(1-z / p)}+\frac{1}{z}(1-P(z)), \quad z \in D .
$$


Now we use the fact that $P$ has the above properties if and only if there exists a function $\psi$ holomorphic in $D$ such that $\psi(D) \subset \bar{D}$ and

$$
P(z)=\frac{1+z\left(\frac{\psi(z)\left(\frac{z-p}{1-z p}\right)+p}{1+\psi(z)\left(\frac{z-p}{1-z p}\right) p}\right)}{1-z\left(\frac{\psi(z)\left(\frac{z-p}{1-z p}\right)+p}{1+\psi(z)\left(\frac{z-p}{1-z p}\right) p}\right)}, \quad z \in D
$$

If we insert (10) into (9), we get

$$
\frac{f^{\prime \prime}(z)}{f^{\prime}(z)}=\frac{2 p}{1-z p}+\frac{2}{p(1-z / p)}+2 \frac{\psi(z)(z-p)+(1-z p) p}{\psi(z)(z-p)^{2}-(1-z p)^{2}} .
$$

The same identity results from (8). This proves the equivalence of (2) and (8). To derive (7) we replace in (11) the function $\psi$ by

$$
\psi(z):=\frac{p^{2}-\omega(z)}{1-\omega(z) p^{2}} .
$$

Obviously, $\omega$ is holomorphic in $D$ and $\omega(D) \subset \bar{D}$, the replacement yields (7) and therefore proves the assertion of Theorem 1.

Theorem 2. Let $f \in \operatorname{Co}(p), p \in(0,1)$ and $n \in\{2,3,4,5\}$. Then the domain of variability of $a_{n}(f)$ is described by the inequality (6). A point on the boundary of this domain is attained if and only if there exists a $\theta \in[0,2 \pi)$ such that $f=f_{\theta}$, where $f_{\theta}$ is defined as in (4).

Proof. The functions

$$
f\left(c_{0} ; z\right)=\frac{z-\frac{p}{1+p^{2}}\left(1+c_{0}\right) z^{2}}{(1-z / p)(1-z p)}, \quad c_{0} \in \bar{D},
$$

belong to $\operatorname{Co}(p)$. This is a consequence of the fact that they satisfy the condition (7) for $\omega(z) \equiv c_{0}$. On the other hand, a computation of the coefficients $a_{n}\left(f\left(c_{0} ; \cdot\right)\right)$ shows that for any point of the domain described by (6) there exists a $c_{0}$ such that $a_{n}(f)=a_{n}\left(f\left(c_{0} ; \cdot\right)\right)$.

A lengthy, but straightforward computation using (7) and the expansion

$$
\omega(z)=\sum_{k=0}^{\infty} c_{k} z^{k}
$$

yields representations for $a_{n}(f)$ of the form

$$
a_{n}(f)=C_{n}(p)-R_{n}(p) c_{0}-T_{n},
$$

where

$$
C_{n}(p)=\frac{1-p^{2 n+2}}{p^{n-1}\left(1-p^{4}\right)}
$$


represent the centers of the domains of variability,

$$
R_{n}(p)=\frac{p^{2}\left(1-p^{2 n-2}\right)}{p^{n-1}\left(1-p^{4}\right)}
$$

their radii, and $T_{n}$ the following remainder terms:

(13) $T_{3}=\frac{\alpha c_{1}}{6}$,

$$
\begin{aligned}
T_{4}= & \frac{1}{24}\left(\alpha^{2} c_{1}+\alpha^{2} c_{0} c_{1}+2 \alpha c_{2}+8 c_{1}\right) \\
T_{5}= & \frac{1}{120}\left(\left(2 \alpha^{3}-10 \alpha+\frac{80}{\alpha}\right) c_{1}+2 \alpha^{3} c_{0}^{2} c_{1}+\left(4 \alpha^{3}+8 \alpha\right) c_{0} c_{1}\right. \\
& \left.+3 \alpha^{2} c_{1}^{2}+\left(4 \alpha^{2}+20\right) c_{2}+4 \alpha^{2} c_{0} c_{2}+6 \alpha c_{3}\right) .
\end{aligned}
$$

Now, we have to prove that for $n \in\{2,3,4,5\}$ the inequality

$$
\left|R_{n}(p) c_{0}+T_{n}\right| \leq R_{n}(p)
$$

is valid for $p \in(0,1)$ and that equality in (16) is attained if and only if $\omega(z) \equiv e^{i \theta}, \theta \in[0,2 \pi)$.

For (12) this is trivial, in the other cases we proceed as follows. We use the triangle inequality to break the left side of (16) into pieces. Then we use the inequalities

$$
\left|c_{n}\right| \leq 1-\left|c_{0}\right|^{2}, \quad n \geq 2,
$$

for the Taylor coefficients of the unimodular bounded function $\omega$. As an abbreviation we use

$$
x:=\left|c_{0}\right|
$$

and we replace any expression in $p$ by the corresponding expression in $\alpha$.

According to (13) we have to prove in the case $n=3$ that

$$
\left|c_{0}+\frac{\alpha}{6} c_{1}\right| \leq 1
$$

This follows in the way explained above from the inequality

$$
(-1+x)\left(1-\frac{\alpha}{6}(1+x)\right) \leq 0
$$

for $x \in[0,1]$ and $\alpha \in(0,1)$. Equality is attained here if and only if $x=1$. This proves Theorem 2 in the case $n=3$.

In the same way we get, for the case $n=4$ from (14) and for the case $n=5$ from (15), an inequality of the form

$$
(-1+x) S_{n}(\alpha, x) \leq 0
$$


that has to be proved for $x \in[0,1]$ and $\alpha \in(0,1)$. In these two cases we show that $S_{n}(\alpha, x)>0$ for $x \in[0,1]$ and $\alpha \in(0,1)$ to get the desired conclusions. For $n=4$ this is a simple task, since

$$
\begin{aligned}
S_{4}(\alpha, x) & =\frac{4-\alpha^{2}}{2 \alpha}-\frac{1}{24}\left(\alpha^{2}+2 \alpha+8+\left(2 \alpha^{2}+2 \alpha+8\right) x+x^{2}\right) \\
& \geq \frac{1}{2 \alpha}\left(4-\alpha^{2}-\frac{\alpha}{12}\left(3 \alpha^{2}+4 \alpha+17\right)\right)>\frac{1}{2 \alpha}>0
\end{aligned}
$$

for $x \in[0,1]$ and $\alpha \in(0,1)$. Hence, (17) is true for $n=4$ and equality is attained there if and only if $x=1$.

For $n=5$ the proof is a bit more complicated. In this case we get

$$
S_{5}(\alpha, x)=\frac{1}{\alpha^{2}}\left(4-2 \alpha^{2}-\frac{\alpha}{120} U(\alpha, x)\right)
$$

where

$$
\begin{aligned}
U(\alpha, x)= & 2 \alpha^{4}+7 \alpha^{3}-4 \alpha^{2}+20 \alpha+80+\left(6 \alpha^{4}+11 \alpha^{3}+4 \alpha^{2}+20 \alpha+80\right) x \\
& +\left(6 \alpha^{4}+\alpha^{3}+8 \alpha^{2}\right) x^{2}+\left(2 \alpha^{4}-3 \alpha^{3}\right) x^{3} .
\end{aligned}
$$

From the inequality

$$
\frac{\partial U(\alpha, x)}{\partial x} \geq 6 \alpha^{4}+11 \alpha^{3}+4 \alpha^{2}+20 \alpha+80+\left(24 \alpha^{4}-7 \alpha^{3}+16 \alpha^{2}\right) x>0
$$

for $x \in[0,1]$ and $\alpha \in(0,1)$ we conclude that

$$
\begin{aligned}
S_{5}(\alpha, x) & \geq S_{5}(\alpha, 1) \\
& =\frac{1}{\alpha^{2}}\left(4-2 \alpha^{2}-\frac{\alpha}{120}\left(16 \alpha^{4}+16 \alpha^{3}+8 \alpha^{2}+40 \alpha+160\right)\right)>0
\end{aligned}
$$

for $x \in[0,1]$ and $\alpha \in(0,1)$. The last inequality implies the truth of (17) in the case $n=5$ and as above we see that equality is attained there if and only if $x=1$. This completes the proof of Theorem 2 .

Acknowledgements. The author thanks F. G. Avkhadiev and Ch. Pommerenke for helpful discussions.

\section{References}

[1] F. G. Avkhadiev, Ch. Pommerenke and K.-J. Wirths, On the coefficients of concave univalent functions, Math. Nachr., to appear.

[2] F. G. Avkhadiev and K.-J. Wirths, Convex holes produce lower bounds for coefficients, Complex Var. 47 (2002), 553-563.

[3] - - - On a conjecture of Livingston, Mathematica (Cluj), to appear.

[4] A. E. Livingston, Convex meromorphic mappings, Ann. Polon. Math. 59 (1994), 275-291.

[5] J. Miller, Convex meromorphic mappings and related functions, Proc. Amer. Math. Soc. 25 (1970), 220-228. 
[6] J. Miller, Convex and starlike meromorphic functions, ibid. 80 (1980), 607-613.

[7] J. Pfaltzgraff and B. Pinchuk, A variational method for classes of meromorphic functions, J. Anal. Math. 24 (1971), 101-150.

Institut für Analysis

TU Braunschweig

38106 Braunschweig, Germany

E-mail: kjwirths@tu-bs.de 